\title{
Using lightweight inference to solve lightweight problems
}

\author{
Joost Vennekens` and Marc Denecker \\ \{joost.vennekens, marc.denecker\}@cs.kuleuven.be \\ Dept. Computerscience, K.U. Leuven \\ Celestijnenlaan 200A \\ B-3001 Heverlee, Belgium
}

Traditionally, Logic Programming and related Non-monotonic Reasoning formalisms have mainly been applied to "hard" AI problems, such as planning, scheduling, constraint solving, belief revision, etc. In the real world of software engineering, however, these hard problems are vastly outnumbered by more mundane tasks. A significant part of software that is written today consists of applications that any reasonably experienced programmer could write in a couple of weeks, using whatever imperative language happens to be the industry standard du jour.

A typical example of this kind of applications is so-called configuration software. The idea is here that there a number of allowable configurations - say of a computer system, or of a server network, or a study program, or a life insurance, or a bicycle, or so on-and that the user must be guided through a number of choices that will eventually result in the configuration that is best suited to his desires. Programming such an application poses no great challenges, neither conceptually nor computationally. The main source of complexity here lies in the domain knowledge concerning valid configurations: this might imply complex dependencies, which could be tedious to encode in an imperative language, especially if the application is also supposed to proactively narrow down the options still available to the user, as more and more choices are made.

While such applications might seem trivial in comparison to the great open problems of AI, we believe that computational logic might also have an interesting role to play here. The goal is in this context not to accomplish the previously impossible, but rather to do existing things better. By leveraging the expressive knowledge representation capabilities of Logic Programming and similar languages, such applications could be written more easily, in less lines of "code", and hopefully considerably quicker - maybe even in days rather than weeks. An additional advantage is that once domain knowledge has been declaratively expressed, it can also be reused for different applications in the same domain. For instance, a specification of the requirements for a schedule of courses at some university might be used at the start of the semester to produce a full schedule, but it might also be used later - by a different UI front-end - to help a secratary adapt the schedule to unforseen circumstances, such as an absent TA or urgent maintanance in some class room.

\footnotetext{
* Joost Vennekens is a post-doctoral researcher of FWO-Vlaanderen.
} 
The setting of configuration software is somewhat different from the setting traditionally considered in LP, though, and therefore it comes with its own set of requirements. For instance, solving a scheduling problem is a task that is typically performed off-line and on a reasonably good machine. Configuration software, on the other hand, is interactive, and might be running on a webserver, together with who knows how many other threads. So, the computational requirements are much more stringent in this case. There is also good news, however, and that is that configuration software does not need to be complete. Because it runs interactively, it is not absolutely necessary that all consequences of a user's choice are immediately shown on-screen; if one is missed at first, it will still eventually be detected after the user has filled in more of the form.

This kind of lightweight interactive applications, therefore, seems to call for anytime algorithms, that may only be approximative and offer no guarantees of completeness, but do run fast in limited memory and will provide useful output even when interrupted before completion. These requirements are rather incompatible with the ground-and-solve approach that most Answer Set Programming systems currently take. An integrated "ground on demand" approach would be much more suited.

In [5], we have presented an algorithm that could work well in such cases. The roots of this algorithm lie in the IDP-system [2]. This is a model expansion system [3] for the language $\mathrm{FO}(\cdot)$, an extension of first-order logic with, among others, a LP-based representation of inductive definitions. IDP is a quite competitive system, which ranked fourth in the global ranking of the second ASP competition [1]. It uses a standard ground-and-solve architecture, in which the grounder already performs a number of logical propagation steps that attempt to minimize the size of the grounding. This propagation algorithm has been extended to a polytime approximate reasoning algorithms for full $\mathrm{FO}(\cdot)$ [5]. In [4], we have used this algorithm to develop an $\mathrm{FO}(\cdot)$ framework for the implementation of configuration software.

Approximation is not the only way of providing inference algorithms that are able to deal with the strict computational requirements of interactive software. In certain contexts, it might also be sometimes possible to exploit computations that have previously been carried out off-line. A prime example is railroad scheduling: the regular timetables are computed far in advance, but if some technical failure occurs, the schedule has to be quickly adapted online, possibly in interaction with an engineer. This can only be accomplished if the existing schedule is leveraged as much as possible and only minimal changes are applied - instead of a "from skratch" model generation system, we therefore need an incremental model revision approach. [?] outlines one such algorithm.

In summary, we believe that, in addition to the traditional "hard" AI problems that have been tackled, there are many "easy" problems in software engineering that could benefit from a declarative LP-style approach. However, this calls for different inference algorithms, which satisfy the requirements of interactiveness by means of anytime approximation, or by leveraging as much as possible the result of computations that were previously performed off-line. The 
development and further refinement of such algorithms is a useful step towards increasing the impact that Logic Programming and other Non-monotonic Reasoning formalisms have on the everyday life of software engineers world-wide.

\section{References}

1. M. Denecker, J. Vennekens, S. Bond, M. Gebser, and M. Truszczyński. The second answer set programming competition. In LPNMR, 2009.

2. M. Mariën, J. Wittocx, and M. Denecker. The IDP framework for declarative problem solving. In $L A S H, 2006$.

3. David G. Mitchell and Eugenia Ternovska. A framework for representing and solving np search problems. In $A A A I$, pages 430-435, 2005.

4. H. Vlaeminck, J. Vennekens, and M. Denecker. A logical framework for configuration software. In PPDP, 2009.

5. J. Wittocx and M. Denecker. Approximate reasoning in first-order logic theories. 2008. 